\title{
Yolk Sac Tumor and Fanconi Anemia: Case Report
}

\author{
Chabchoub Imen1, Sboui Hiba1*, Ammar Nouha1, Ben Abdeljalil Nouha², Hochlef Makrem1, \\ Belhassen Samia ${ }^{3}$, Zrig Ahmed ${ }^{4}$, Zakhama Abdelfattah ${ }^{2}$, Chabchoub Elyes ${ }^{5}$, Ben Ahmed Slim 1
}

${ }^{1}$ Department of Medical Onocology, Faculty of Medecine Sousse, University Hospital Farhat Hached, Sousse, Tunisia ${ }^{2}$ Department of Anathomopathology, Faculty of Medecine Monastir, University Hospital Fatouma Bourguiba, Monastir, Tunisia ${ }^{3}$ Department of Pediatric Surgery, Faculty of Medecine Monastir, University Hospital Fatouma Bourguiba, Monastir, Tunisia ${ }^{4}$ Department of Radiology, Faculty of Medecine Monastir, University Hospital Fatouma Bourguiba, Monastir, Tunisia ${ }^{5}$ Department of Genetics, Faculty of Medecine Sousse, University Hospital Farhat Hached, Sousse, Tunisia Email: *sboui-hiba2006@hotmail.com

How to cite this paper: Imen, C., Hiba, S., Nouha, A., Nouha, B.A., Makrem, H., Samia, B., Ahmed, Z., Abdelfattah, Z., Elyes, C. and Slim, B.A. (2021) Yolk Sac Tumor and Fanconi Anemia: Case Report. Journal of Cancer Therapy, 12, 585-592. https://doi.org/10.4236/jct.2021.1210050

Received: September 19, 2021

Accepted: October 26, 2021

Published: October 29, 2021

Copyright $\odot 2021$ by author(s) and Scientific Research Publishing Inc. This work is licensed under the Creative Commons Attribution International License (CC BY 4.0).

http://creativecommons.org/licenses/by/4.0/

\begin{abstract}
Yolk sac tumor (YST), which most frequently arises in the gonads as a type of germ cell tumor, is rare in children but is highly malignant. The common age group affected by yolk sac tumours is 11 to 24 years. In this study, we described a rare case of YST in terms of the clinical manifestation, imaging, and histopathology findings, diagnosis and treatment in a 12-year-old girl who was followed since birth for Fanconi-like syndrome. This is a rare clinical association that also limits chemotherapy.
\end{abstract}

\section{Keywords}

Alpha-Fetoprotein, Chemotherapy, Yolk Sac Tumor, Pediatric, Surgery, Fanconi-Like Syndrome

\section{Introduction}

The association of malformative abnormalities and cancer is known in several situations: such as Wiedeman-Beckwith syndrome, neurofibromatosis type 1, defect in DNA repair, Drash syndrome and WAGAR syndrome Wilms tumor, aniridia, genitourinary anomalies, and intellectual disability genitourinary anomalies, and intellectual disability). On the other hand, several other associations remain unexplained, and the fortuitous or significant character is not always obvious.

Fanconi-like syndrome is a genetic abnormality characterized by chromosomal instability responsible for the malformation syndrome. And it is a syndrome 
predisposing to leukemia.

Germ cell tumors represent a group of tumors heterogeneous histologically and clinically. They account for about $3 \%$ of childhood and adolescent cancers. Epidemiologically, they have a bimodal distribution with the first peak in infancy and a second larger peak during the peripubertal period. We distinguish histologically:

- Seminomatous malignant germ tumors (dysgerminoma).

- Non-seminomatous malignant germ tumors, of which there are four types: yolk sac tumor, choriocarcinoma, embryonic carcinoma and immature teratoma.

Ovarian vitelline tumors also called endodermal sinus tumors, or YST, are very rare. They represent $2 \%-5 \%$ of all ovarian tumors and less than $5 \%$ of all germ-tumors. AFP is a very sensitive marker whose rate is correlated with tumor volume [1]. These tumors are readily chemosensitive and the treatment may be conservative. We present the case of an 11-year-old girl followed since birth for Fanconi-like syndrome and growth retardation, who has been followed since April 2018 for a vitelline tumor of the ovary.

\section{Observation}

This is a 12-year-old girl, who has a malformative syndrome. The karyotype made in the framework of exploration of its disease, objectified the presence of a chromosomal instability syndrome with $39 \%$ of chromosomal breaks.

The patient was consulted in the emergency room for abdominal pain and vomiting. The examination showed stunting with a weight $=18 \mathrm{~kg}$, a size $=120$ $\mathrm{cm}$, microcephaly, fever $=38^{\circ}$, a flexible depressible abdomen with the presence of a palpable mass at the level of the right iliac fossa lasts $8 \mathrm{~cm}$ whose limits are not clear, fixed, painful without inflammatory signs opposite.

The abdominopelvic ultrasonography showed a $93 \times 70 \mathrm{~mm}$ abdominal-pelvic mass of solid heterogeneous structure containing several fluid chambers with anechoic content, probably of right adnexal origin.

The abdominopelvic CT showed the presence of a large pre-rectal medial pelvic mass measuring $10 \times 7 \times 6 \mathrm{~cm}$ pushing the bladder forward hupodense double component: a tense majority cystic, having a thin wall enhanced by PDC injection, with exo vasiculation anterior measuring $37 \times 17 \mathrm{~mm}$, and a heterogeneous solid enhanced after injection of PDC, with parietal calcifications (Figure 1).

The biological assessment was disrupted with a very high AFP level at 36352 $\mathrm{ng} / \mathrm{ml}$. Biologic markers CA125 and beta HCG values were negative.

The patient was operated on. She presented a vascularized mass, at the expense of the left ovary but lateralised on the right, adhering to the posterior wall and to the anterior surface of the rectum and infiltrating part of the epiploon as well as the appendiceal tip. She underwent a left annexectomy, appendectomy and resection of the omentum and then an abundant wash (Figure 2 and Figure 3). 


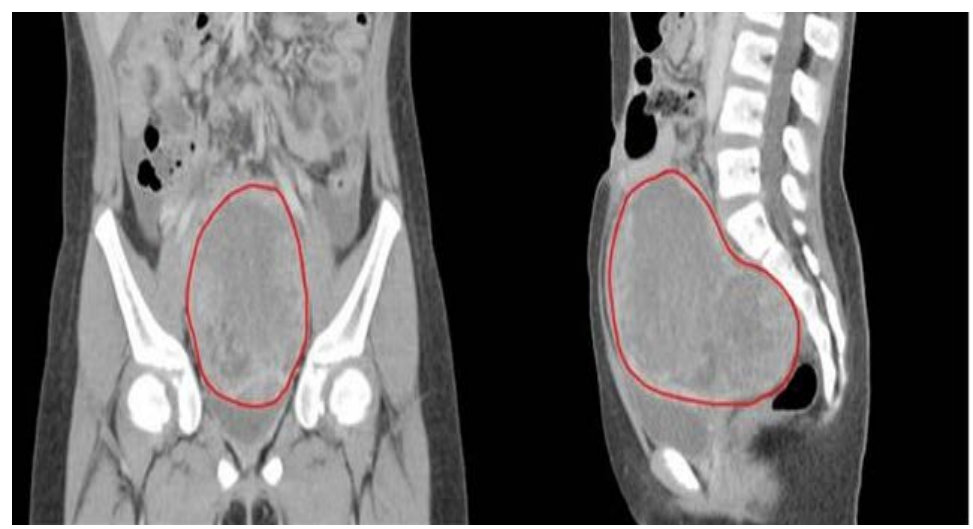

Figure 1. A large pre-rectal medial pelvic mass measuring $10 \times 7 \times 6 \mathrm{~cm}$.

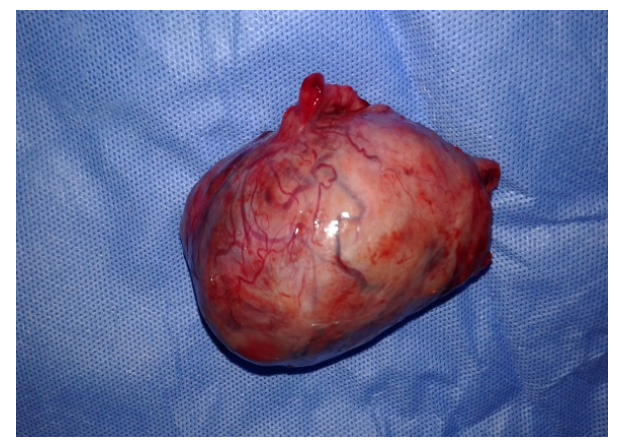

Figure 2. A piece of left annexcectomy measuring $10 \times 6.5 \times 5.5 \mathrm{~cm}$.

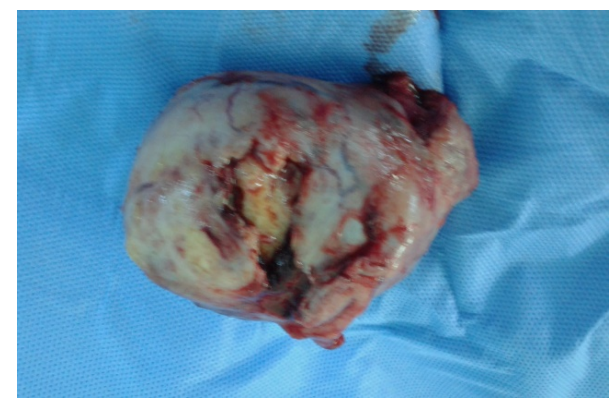

Figure 3. A piece of left annexectomy.

The final pathological examination showed a piece of left anexectomy formed by the ovary measuring $10 \times 6,5 \times 5.5$. The trunk is $3.5 \mathrm{~cm}$ long and $0.5 \mathrm{~cm}$ in diameter. At the cut, the ovary is totally occupied by the tumor in hemorrhagic changes. On microscopy, the tumor is necrotic and shows a lobulated appearance. It is formed by microcystic structures lined with tumor cells of epithelial appearance of variable size, with clear cytoplasm sometimes reshaping a cytoplasmic vacuole with hyaline lobules. The nucleus is moderately atypical with irregular contours, often nucleolar and shows many figures of mitosis. There are 20 mitoses per 10 fields. The tumor cells often cover the papillary structures with central connective axis, these structures are visible in the light of a cystic cavity producing Schiller Duval bodies. The tumor is the site of edematous and hemorrhagic changes and is traversed by fibrous septa (Figure 4, Figure 5). 


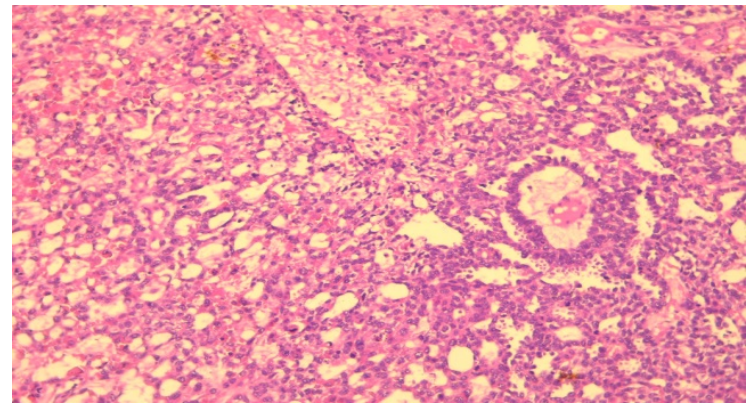

Figure 4. Microcystic pattern with schiller-Duval bodies $\mathrm{HE} \times 100$.

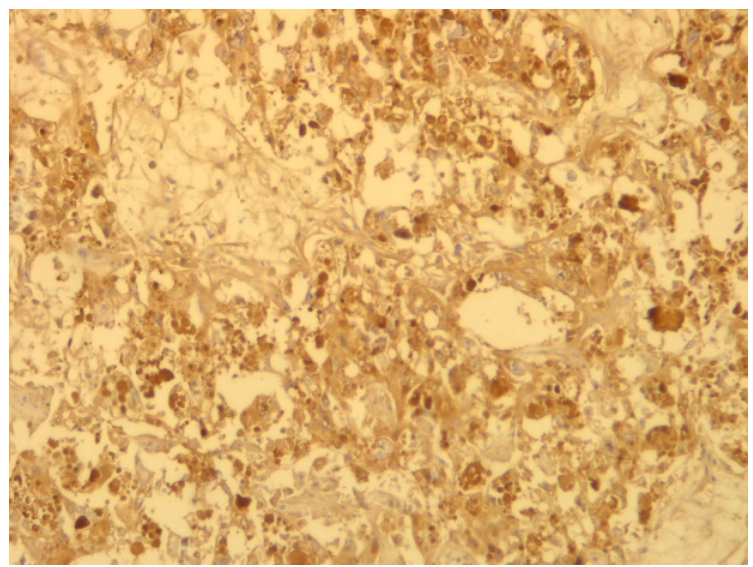

Figure 5. The Immunostaining showing diffuse positivity for AFP.

Therefore, a vitelline tumor (yolk sac tumor or endodermal sinus tumor) was found in the left ovary. On day 7, the rate of AFP was 7,882 UI with a negative rate of HCG.

Four chemotherapy courses based on cisplatin $\left(20 \mathrm{mg} / \mathrm{m}^{2}\right.$ daily from day 1 to day 5), etoposide ( $75 \mathrm{mg} / \mathrm{m}^{2}$ daily from day 1 to day 5) and ifosfamide $\left(3 \mathrm{~g} / \mathrm{m}^{2}\right.$ daily, day 1 and day 2) were established, according to the TGM 95 protocol of the French Society of Pediatric Oncology (SFOP). The patient presented episodes of febrile neutropenia that require hospitalization. These hematologic toxicities were well curbed.

The patient was re-examined 1 month after surgery. An abdominal and pulmonary CT found no recurrence or metastasis. The patient is incomplete remission since 3 years.

\section{Discussion}

Childhood cancers are rare diseases that account for only $1 \%$ of all human cancers [2]. On the other hand, their possible genetic origin has been studied extensively and some tumors have been used as models for understanding the mechanisms of carcinogenesis. The syndromes of predisposition are thus very varied that rare. Less than $5 \%$ of children with cancer is a transmitted genetic mechanism.

Genetic factors are of 3 types: 
- Gene, disease derived from a single gene mutation;

- Chromosomes with genetic imbalance by default or excess of chromosomal material;

- Polygenic or multifactorial.

Among the syndromes of predisposition to cancer: we have anemia of Fanconi. It is a rare human genetic syndrome with recessive inheritance characterized by an extremely complex and heterogeneous phenotype. The main clinical manifestation of AF is the presence of cytopenia that can affect all cellular elements of the blood (pancytopenia). This peripheral abnormality correlates with the presence of a hypoplastic marrow. AF patients may also have several very different congenital malformations: growth retardation, abnormalities of the thumb (which may be absent, hypoplastic or supernumerary), reduced fertility, coffee-milk spots, kidney abnormalities, microcephaly, microphthalmia, abnormalities of the Digestive. Nevertheless, the interindividual variation is very important, with individuals presenting only minor and non-disabling abnormalities and others characterized by a very serious clinical attack [2] [3] [4].

It should be noted that, although the clinical phenotype of AF is highly variable, two serum characteristics are present in almost all patients: a high titer of $\alpha$-fetoprotein and a very high titer of TNF- $\alpha$, a cytokine pro-inflammatory and cytotoxic.

$\mathrm{AF}$ is associated with a very high risk of developing cancer, it is estimated that about $30 \%$ of patients will develop a tumor during their lifetime: acute myeloid leukemia ( $15 \%$ - $20 \%$ of patients), a cancer of the oral cavity or hepatocarcinoma ( $5 \%$ of patients). In our case, the patient developed a vitelline tumor or yolk Tumor (YT). To our knowledge, this is the first case of YT reported in the literature. Germ cell tumors represent a group of tumors heterogeneous histologically and clinically. They account for about 3\% of childhood and adolescent cancers. Epidemiologically, they have a bimodal distribution with the first peak in infancy and a second larger peak during the peripubertal period. We distinguish: 1) Seminomatous malignant germ tumors (dysgerminoma); 2) Non-seminomatous malignant germ tumors, of which there are four types: yolk sac tumor, choriocarcinoma, embryonic carcinoma and immature teratoma.

The YT represent the most frequent histological subtype before 10-year-old. They are defined microscopically by the presence of Schiller-Duval bodies (central vessel bordered by 2 rows of tumor cells separated by a free interval) and labeled with AFP in IHC.

Abdominal pain and pelvic mass palpation are the most common signs $(85 \%$ of cases) [5]. This type of tumor may be revealed by ascites or peritonitis secondary to twisting or infection [6]. In the case of our patient, the tumor was revealed by intense pelvic pain. The radiological diagnosis of vitelline tumors is difficult [7]. Several studies, including abdominal and transvaginal ultrasonography, CT and MRI, have shown that the tumor often has a large, hypervasculated, massive appearance that may be associated with a cystic component and haemorrhagic foci [7]. However, none of these examinations seems to be specific 
for this type of tumor [7]. In the case of our patient, the cystic part was predominant with vascularized septa. The diagnosis is mainly based on the histological examination and the biological assays of the tumor markers (in particular AFP). Indeed, biology benefits from the contribution of AFP, which is a very sensitive marker for this tumor. It can be detected in the blood but also on the histological sections of the tumor by the immunohistochemistry technique. A very high value of AFP can strongly guide the diagnosis [8]. It is also a sensitive marker for monitoring evolution [9]. A very rapid fall postoperatively is a good sign in favor of the absence of tumor residue [10]. The effectiveness of chemotherapy is also judged on the standardization of AFP [5]. In our observation, the AFP value dropped from 36,352 to $7882 \mathrm{ng} / \mathrm{ml}$ in the immediate postoperative period, suggesting no tumor residues. It has been shown that any recurrence is early detected by the elevation of AFP [9].

Standard treatment consists of surgery associated with chemotherapy. This attitude is related to the significant risk of recurrence of yolk sac tumors in the absence of adjuvant chemotherapy [3].

Adjuvant chemotherapy reduces the risk of recurrence [8] [9]. The tumor is especially sensitive to platinum sels.

SFOP, in its TGM 95 protocol, recommends chemotherapy which differs according to the prognostic group of the germinal tumor and defines two forms:

- Standard forms of risk: initial AFP less than 15,000 and no metastases. For whom the recommended protocol is VBP (vinblastine $3 \mathrm{mg} / \mathrm{m}^{2}$ daily, day 1 and day 2, bleomycin $15 \mathrm{mg} / \mathrm{m}^{2}$, day 1 and day 2, cisplatin $100 \mathrm{mg} / \mathrm{m}^{2}$ day 3);

- High risk forms: initial AFP > 15,000 and/or presence of diagnostic metastases.

The recommended protocol, in this case, is VIP (ifosfamide $3 \mathrm{~g} / \mathrm{m}^{2}$ daily, day 1 and day 2 , etoposide $75 \mathrm{mg} / \mathrm{m}^{2}$ daily, day 1 to day 5 , cisplatin $20 \mathrm{mg} / \mathrm{m}^{2}$ daily, day 1 to day 5 ).

Given the young age of our patient, it was treated according to the protocol of the SFOP. She was classified in the high-risk group with very high initial AFP and therefore had four courses of chemotherapy using the VIP protocol. Several prognostic factors of vitelline tumors are reported in the literature:

- Advanced tumor stage, postoperative tumor residue, ascites of high abundance are factors of poor prognosis [5]. Five-year survival varies in the literature between $42 \%$ and $74 \%$ if the ascites are greater than $100 \mathrm{ml}$ and between $58 \%$ and $86 \%$ with a volume of ascites greater than $100 \mathrm{ml} \mathrm{[3]} \mathrm{[5].}$

- The combination of cisplatin in the chemotherapy regimen improves survival [5].

- The prognostic value of the initial AFP level is very controversial in the literature [7] [8] [10].

The follow up of children treated for a vitelline tumor, apart from the usual elements of ovarian carcinoma fellow up, uses the AFP assay. The recommended postoperative monitoring rhythm is as follows [5]:

- A clinical examination: monthly during the first year, fortnightly the second 
year, quarterly the third, every four months during the fourth year and then every six months until ten years.

- A dosage of AFPs: every two weeks for six months, then every month until the 12th month, a monthly dosage the second year, each trimester the third year, every four months the fourth year, then every six months until ten years.

- An abdominopelvic scan: three to six weeks after surgery, then at three months.

At the last follow-up of one year, our patient is in complete remission. She had a CT exam at 6 months that did not show recurrence, her AFP rate is still normal.

\section{Conclusions}

This rare case showed that Fanconi anemia can be limiting for chemotherapy but does not contraindicate it given the aggressiveness of the yolk tumors.

There are several predisposing syndromes to cancer, which are characterized by chromosomal instability that can promote the onset of cancer. Understanding the mechanism responsible for this phenomenon would improve future cancer therapies.

\section{Conflicts of Interest}

The authors declare no conflicts of interest regarding the publication of this paper.

\section{References}

[1] Dällenbach, P., Bonnefoi, H., Pelte, M.F. and Vlastos, G. (2006) Yolk Sac Tumours of the Ovary: An Update. European Journal of Surgical Oncology, 32, 1063-1075. https://doi.org/10.1016/j.ejso.2006.07.010

[2] Fernandes, P. (2018) The Roles of FANCD2 in the Maintenance of Common Fragile Site Stability. Université Paris Saclay (COmUE), Paris.

[3] Macé, G., Briot, D., Guervilly, J.-H. and Rosselli, F. (2007) Fanconi Anemia: Cellular and Molecular Features. Pathologie Biologie, 55, 19-28.

https://doi.org/10.1016/j.patbio.2006.04.008

[4] Gueye, A., Narducci, F., Baranzelli, M.C., et al. (2007) Malignant Ovarian Germ Cell Tumours: A Trial of 36 Cases. Gynécologie Obstétrique \& Fertilité, 35, 406-419. https://doi.org/10.1016/j.gyobfe.2007.01.028

[5] Talerman, A. (1994) Germ Cell Tumors of the Ovary. In: Kurman, R.J., Ed., Blaustein's Pathology of the Female Genital Tract, 4th Edition, Springer-Verlag, New York, 849-914. https://doi.org/10.1007/978-1-4757-3889-6_20

[6] Kawai, M., Kano, T., Furuhashi, Y., et al. (1991) Prognostic Factors in Yolk Sac Tumors of the Ovary. A Clinicopathologic Analysis of 29 Cases. Cancer, 67, 184-192. https://doi.org/10.1002/1097-0142(19910101)67:1<184::AID-CNCR2820670131>3.0 .CO;2-C

[7] Guastalla, J.P. and Ray-Coquard, I. (2006) Les cancers ovariens dans: Fl'chon A. et Droz JP. Biologie des tumeurs germinales. SpringerVerlag, Paris, 477-495. 
[8] Tewari, K., Cappuccini, F., Disaia, P.J., et al. (2000) Malignant Germ Cell Tumors of the Ovary. Obstetrics \& Gynecology, 95, 128-133.

https://doi.org/10.1097/00006250-200001000-00024

[9] Mitchell, P.L., Al-Nasiri, N., A’Hern, R., et al. (1999) Treatment of Nondysgerminomatous Ovarian Germ Cell Tumors: An Analysis of 69 Cases. Cancer, 85, 2232-2244.

https://doi.org/10.1002/(SICI)1097-0142(19990515)85:10<2232::AID-CNCR19>3.0. $\mathrm{CO} ; 2-4$

[10] Talerman, A., Haije, W.G. and Baggerman, L. (1978) Serum Alphafetoprotein (AFP) in Diagnosis and Management of Endodermal Sinus (Yolk SAC) Tumor and Mixed Germ Cell Tumor of the Ovary. Cancer, 41, 272-278.

https://doi.org/10.1002/1097-0142(197801)41:1<272::AID-CNCR2820410138>3.0.C $\underline{\mathrm{O} ; 2-\mathrm{Z}}$ 